\title{
Duplicated Vertebral Artery : Literature Review and Clinical Significance
}

\author{
Myoung Soo Kim, M.D., Ph.D. \\ National Medical Center, Seoul, Korea
}

Objective : Duplication of the vertebral artery (VA) is a rare vascular variant. This paper describes the anatomy and embryological development of duplicated VAs and reviews the clinical significance.

Methods : Computed tomography (CT) angiography was performed in 3386 patients (1880 females, 1506 males) between March 2014 and November 2015. We defined duplication of the VA as a condition in which the VA has two origins that fused at different levels of the neck.

Results : Ten of the 3386 patients $(0.295 \%)$ who received CT angiography had a dual origin of the VA; three on the left side, and seven on the right side. In all seven with right dual origin of the VA, both limbs of the VA origin originated from the right subclavian artery. In all three patients with left dual origin of the VA, both limbs of the VA originated from the left subclavian artery and aortic arch. In all 10 patients, the medial limb of the duplicated VA was located posteriorly and medially to the common carotid artery (CCA) and anteriorly and laterally to the vertebral transverse foramen. In two patients, the medial limb of the duplicated VA was located in close proximity to the CCA. In another two patients, the medial limb of the duplicated VA was located in close proximity to the CCA, carotid bifurcation, and proximal internal carotid artery.

Conclusion : Although duplication of the VA is asymptomatic in most patients, clinicians should consider this anomaly during diagnosis and treatment.

Key Words : Vertebral artery · Congenital abnormalities · Embryonic development · Computed tomography angiography.

\section{INTRODUCTION}

Duplication of the vertebral artery (VA) is defined as a VA that has two origins, a variable course, and a fusion level in the neck. Duplication of the VA is rarely reported in the literature and has been detected as an incidental finding in autopsy series, in angiographic studies, and in computed tomography (CT) angiography and magnetic resonance angiography ${ }^{5,10,14,17,18)}$. Knowledge of these variants may be important, especially when choosing the right procedure to avoid misinterpretation or injury of a duplicated VA before vascular head and neck surgery, spinal surgery, or cerebral angiography.

Here, I report 10 cases of a duplicated VA that were identified incidentally by CT angiography. This paper describes the anatomy and embryological development of duplicated VAs and reviews the clinical significance of this anomaly.

- Received : February 16, 2017 •Revised : April 7, 2017 •Accepted : April 26, 2017

- Address for reprints : Myoung Soo Kim, M.D., Ph.D.

National Medical Center, Euljiro 245, Jung-gu, Seoul 04564, Korea

Tel : +82-2-2260-7180, Fax : +82-2-2262-4876, E-mail : hanibalkms@hanmail.net

This is an Open Access article distributed under the terms of the Creative Commons Attribution Non-Commercial License (http://creativecommons.org/licenses/by-nc/4.0) which permits unrestricted non-commercial use, distribution, and reproduction in any medium, provided the original work is properly cited. 


\section{MATERIALS AND METHODS}

Images obtained by CT angiography were analyzed for the presence of dual origin of the VA. Duplication of the VA was defined as the presence of two origins of the VA that had fused at different levels of the neck ${ }^{5)}$. CT angiography performed on foreign patients or obtained outside a hospital was excluded. The CT angiographic studies were performed for a variety of clinical reasons, including symptoms of cerebral ischemia, hemorrhagic contusion, intracerebral hemorrhage, headache, dizziness, sensory changes, and routine check-up. All images were evaluated by one neurosurgeon.

CT angiography of the intracranial and extracranial vessels was performed in 3386 patients (1880 females, 1506 males; 58.73 \pm 15.57 , age range 11-94 years) between March 3, 2014, and November 12, 2015, and the results were analyzed. An Aquilion Prime 160-slice CT scanner (Toshiba Medical Systems Corp., Otawara, Japan) was used in 2454 patients, and an Aquilion CXL edition 128-slice CT scanner (Toshiba Medical Systems Corp.) was used in 932 patients. After acquisition of the nonenhanced CT data, contrast-enhanced CT angiography was performed. The parameters for CT angiographic acquisition were as follows. For the Aquilion Prime : $100 \mathrm{kVp}$, $225 \mathrm{mAs}$, field of view $220 \mathrm{~mm}$, detector collimation $80 \times 0.5$ $\mathrm{mm}$, table speed $25.5 \mathrm{~mm} /$ rotation, gantry rotation speed 0.75 s/rotation, reconstructed section thickness $0.5 \mathrm{~mm}$, and reconstruction increment $0.3 \mathrm{~mm}$. For the Aquilion CXL : 120
$\mathrm{kVp}, 250 \mathrm{mAs}$, field of view $240 \mathrm{~mm}$, detector collimation $64 \times 0.5 \mathrm{~mm}$, table speed $20.5 \mathrm{~mm}$ /rotation, gantry rotation speed 0.5 s/rotation, reconstructed section thickness $0.5 \mathrm{~mm}$, and reconstruction increment $0.5 \mathrm{~mm}$. The scan range extended from $2 \mathrm{~cm}$ below the aortic arch to a point $1 \mathrm{~cm}$ above the level of the lateral ventricles.

CT angiography was performed, and the results were analyzed using the following method. A total of $100 \mathrm{~mL}$ of iopamidol (Pamiray ${ }^{\circledR} 370$; Dongkook Pharmaceuticals Co., Seoul, Korea) was administered intravenously using a power injector at a rate of $4.0 \mathrm{~mL} / \mathrm{s}$ via an 18-gauge catheter positioned in a peripheral vein, and the scan delay was individually adapted using a bolus-tracking technique. For the bolus tracking, first, a single nonenhanced low-dose scan at the level of the upper neck was obtained. With the start of contrast material administration, repeated low-dose monitoring scans were obtained every second. When the first contrast was seen in the common carotid artery (CCA), the CT angiography was triggered automatically without any time delay. The data were transferred to a personal computer. Three dimensional reconstructions of the images were performed using commercially available software (Vitrea 2; Vital Images, Minnetonka, MN, USA). From the data, 3D CT angiography images were reconstructed using a volume-rendering technique. A series of 17 projection images at every $20^{\circ}$ around the cephalocaudal axis were generated and then transferred to the picture archiving and communication system.

Table 1. Characteristics of 10 patients with duplicated VA

\begin{tabular}{|c|c|c|c|c|c|c|}
\hline $\begin{array}{l}\text { Case } \\
\text { number }\end{array}$ & Sex & Age & $\begin{array}{c}\text { Side of } \\
\text { duplicated VA }\end{array}$ & $\begin{array}{l}\text { Level of vertebral foramen } \\
\text { entering VA (RT side) }\end{array}$ & $\begin{array}{l}\text { Level of vertebral foramen } \\
\text { entering VA (LT side) }\end{array}$ & $\begin{array}{l}\text { Distance from CCA \& } \\
\text { prxoximal ICA }\end{array}$ \\
\hline 1 & $\mathrm{~F}$ & 34 & LT & C6 & $\mathrm{C} 6, \mathrm{C} 4$ & Close with CCA \\
\hline 2 & M & 35 & LT & C6 & $C 6, C 5$ & Distant from CCA \& ICA \\
\hline 3 & $\mathrm{~F}$ & 38 & RT & $\mathrm{C} 6, \mathrm{C} 4$ & $\mathrm{C} 5$ & Close with CCA \& ICA \\
\hline 4 & $\mathrm{~F}$ & 49 & RT & $\mathrm{C} 6, \mathrm{C} 4$ & $\mathrm{C} 6$ & Distant from CCA \& ICA \\
\hline 5 & $\mathrm{~F}$ & 56 & RT & $\mathrm{C} 6, \mathrm{C} 3$ & $\mathrm{C} 5$ & Close with CCA \& ICA \\
\hline 6 & $\mathrm{~F}$ & 58 & LT & $\mathrm{C} 6$ & $\mathrm{C} 6, \mathrm{C} 5$ & Distant from CCA \& ICA \\
\hline 7 & $\mathrm{~F}$ & 58 & RT & $\mathrm{C} 6, \mathrm{C} 4$ & C6 & Close with CCA \\
\hline 8 & $\mathrm{~F}$ & 64 & RT & $\mathrm{C} 5, \mathrm{C} 3$ & $\mathrm{C} 6$ & Distant from CCA \& ICA \\
\hline 9 & M & 74 & RT & $\mathrm{C} 6, \mathrm{C} 5$ & C6 & Distant from CCA \& ICA \\
\hline 10 & $\mathrm{~F}$ & 75 & RT & $\mathrm{C} 5, \mathrm{C} 4$ & C5 & Distant from CCA \& ICA \\
\hline
\end{tabular}

VA : vertebral artery, RT : right, LT : left, CCA : common carotid artery, ICA : internal carotid artery, F : female, $\mathrm{M}$ : male 


\section{RESULTS}

Ten of the 3386 patients $(0.295 \%)$ who received CT angiography had a dual origin of the VA. Their age range was 34-75 years, and eight were females and two were males. Seven patients had a right dual origin of the VA, and three patients had a left dual origin of the VA (Table 1). In all seven patients with a right dual origin of the VA, both limbs of the VA origin originated from the right subclavian artery. In all three patients with a left dual origin of the VA, both limbs of the VA originated from the left subclavian artery and aortic arch. All 10 patients had no clinical symptoms associated with the dual origin of the VA. Associated vascular variations included one basilar artery fenestration and two left VA origins from the aortic arch.

In all 10 patients, axial CT demonstrated that the medial limb of the duplicated VA was located posteriorly and medially to the CCA and anteriorly and laterally to the vertebral transverse foramen. The medial limb of the duplicated VA was located between the anterior scalene muscle and longus coli muscle in all 10 patients.

In two patients, sagittal CT revealed that the medial limb of the duplicated VA was located in close proximity to the CCA. In another two patients, sagittal CT showed that the medial limb of the duplicated VA was located in close proximity to the CCA, carotid bifurcation, and proximal internal carotid artery (Table 1).

\section{Case 2}

A 35-year-old man with left occipital pain underwent CT angiography. Reconstructed CT angiography showed a left duplicated VA originating from the left subclavian artery and aortic arch. Axial CT demonstrated that the medial limb of the duplicated VA was located anteriorly to the vertebral transverse foramen and medially to the CCA (Fig. 1). The patient's symptom was relieved after conservative management.

\section{Case 3}

A 38-year-old woman was referred to our department because of dizziness. CT angiography demonstrated an incidental right duplicated VA originating from the right subclavian artery. Axial CT showed that the medial limb of the duplicated VA was located posteromedially to the right CCA and an- teriorly to the vertebral transverse foramen. Sagittal CT revealed that the medial limb of the duplicated VA was located in close proximity to the CCA and internal carotid artery (Fig. 2). The patient was managed conservatively, and her dizziness improved.

\section{Case 7}

A 58-year-old woman visited our department for paresthesia of the left occipital area and right arm, and intermittent weakness in both legs. CT angiography showed a right duplicated VA originating from the right subclavian artery. Axial CT demonstrated that the medial limb of the duplicated VA was located posteriorly to the right CCA and anteriorly to the vertebral transverse foramen. Sagittal CT revealed that the medial limb of the duplicated VA was located in close proximity to the CCA (Fig. 3). The patient was managed conservatively, and her clinical symptoms improved.

\section{DISCUSSION}

\section{Embryological review of the duplicated origin of the VA}

During embryological development, the VA is formed by
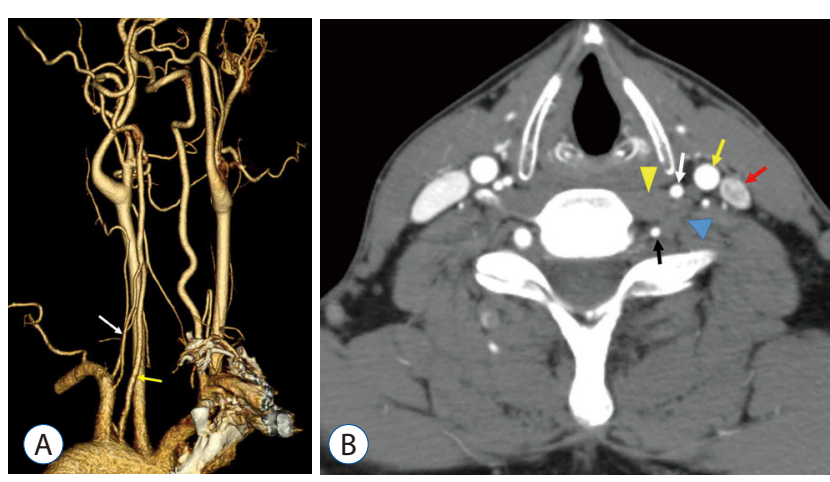

Fig. 1. Reconstructed computed tomographic (CT) angiography shows a left duplicated vertebral artery (VA) originating from the left subclavian artery and aortic arch (A : yellow arrow, medial limb of the left duplicated VA; white arrow, lateral limb of the left duplicated VA). Axial neck CT at the sixth cervical vertebral body level after contrast enhancement shows that the medial limb of the left duplicated VA is located anteriorly to the vertebral transverse foramen. The medial limb of the duplicated VA is located between the longus coli muscle and anterior scalene muscle (B: black arrow, lateral limb of the left duplicated VA; white arrow, medial limb of left VA, red arrow, left internal jugular vein; yellow arrow, left common carotid artery; yellow arrowhead, longus coli muscle; blue arrowhead, anterior scalene muscle). 

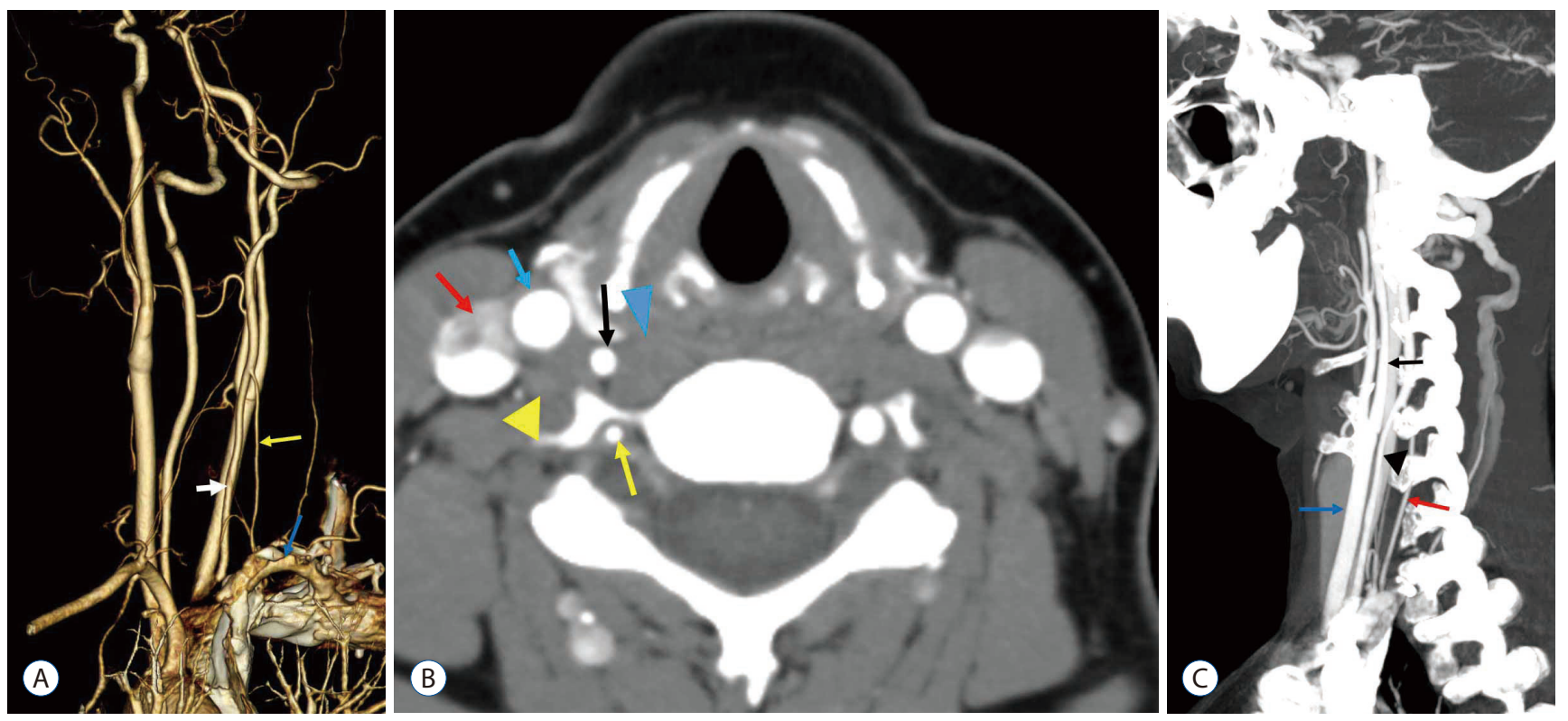

Fig. 2. Computed tomographic (CT) angiography of a 38-year-old woman with a right duplicated vertebral artery (VA) originating from the right subclavian artery. Reconstructed CT angiography shows a right duplicated VA originating from the right subclavian artery (A : blue arrow, right subclavian artery; yellow arrow, lateral limb of the right duplicated VA; white arrow, medial limb of the right duplicated VA). Axial neck CT at the fifth cervical vertebral body level after contrast enhancement shows that the medial limb of the duplicated right VA is located anteriorly to the vertebral transverse foramen and between the longus coli muscle and anterior scalene muscle (B : black arrow, medial limb of the right duplicated VA; yellow arrow, lateral limb of the right VA; blue arrow, right common carotid artery [CCA]; red arrow, right internal jugular vein; blue arrowhead, longus coli muscle; yellow arrowhead, anterior scalene muscle). Sagittal CT after contrast enhancement shows that the medial limb of the duplicated VA is located in close proximity to the CCA and proximal internal carotid artery (C : black arrowhead, medial limb of the duplicated VA; blue arrow, CCA; red arrow, lateral limb of the duplicated VA; black arrow, internal carotid artery).

fusion of the longitudinal anastomoses of the cervical intersegmental arteries, which branch off the primitive paired dorsal aorta. In normal situations, almost all intersegmental arteries regress, with the exception of the seventh intersegmental artery (i.e., the sixth cervical intersegmental artery), which forms the proximal portion of the subclavian artery ${ }^{15)}$. Several mechanisms that may give rise to a separated origin of the VA have been proposed. One explanation suggests that the primitive dorsal aorta does not regress together with the two intersegmental arteries that connect to the VA. This arrangement may give rise to duplication of the $\mathrm{VA}^{16,19)}$. Another possibility is failure of the regression of the fourth, fifth, or sixth intersegmental arteries (i.e., the third, fourth, or fifth cervical intersegmental arteries) ${ }^{4-6)}$. This adds an additional origin to the VA in addition to the normal seventh intersegmental artery. Persistence of the left fourth or fifth (or less frequently third) cervical intersegmental artery may result in an aortic origin or dual origin of the left VA.

During duplication of the VA, one limb can originate from the subclavian artery, whereas the second limb can originate from the aortic arch, subclavian artery, thyrocervical trunk, or innominate trunk $k^{4,12)}$. Rarely, both limbs of a duplicated left VA originate from the aorta ${ }^{8)}$. The left fourth and fifth intersegmental arteries rarely branch off from a stem artery, which originates from the aorta between the left CCA and left subclavian artery. Usually, the medial limb enters the higher vertebral transverse foramen, which is consistent with the theory of intersegmental vessel regression failure, and results from intersegmental arteries that accompany the cervical nerve roots $^{21)}$. In all 10 cases reported here, the medial limb of the duplicated VA entered the higher vertebral transverse foramen.

\section{Clinical implications of a duplicated VA reported in the literature}

Duplication of the origin of the VA has no clinical expression in most cases. I reviewed the reports of symptomatic cases of a duplicated VA and their clinical implications for diagnosis and treatment.

Only two cases of duplication of the VA associated with dissection have been reported ${ }^{1,11)}$. Dare et al. ${ }^{1)}$ reported a 38-year- 

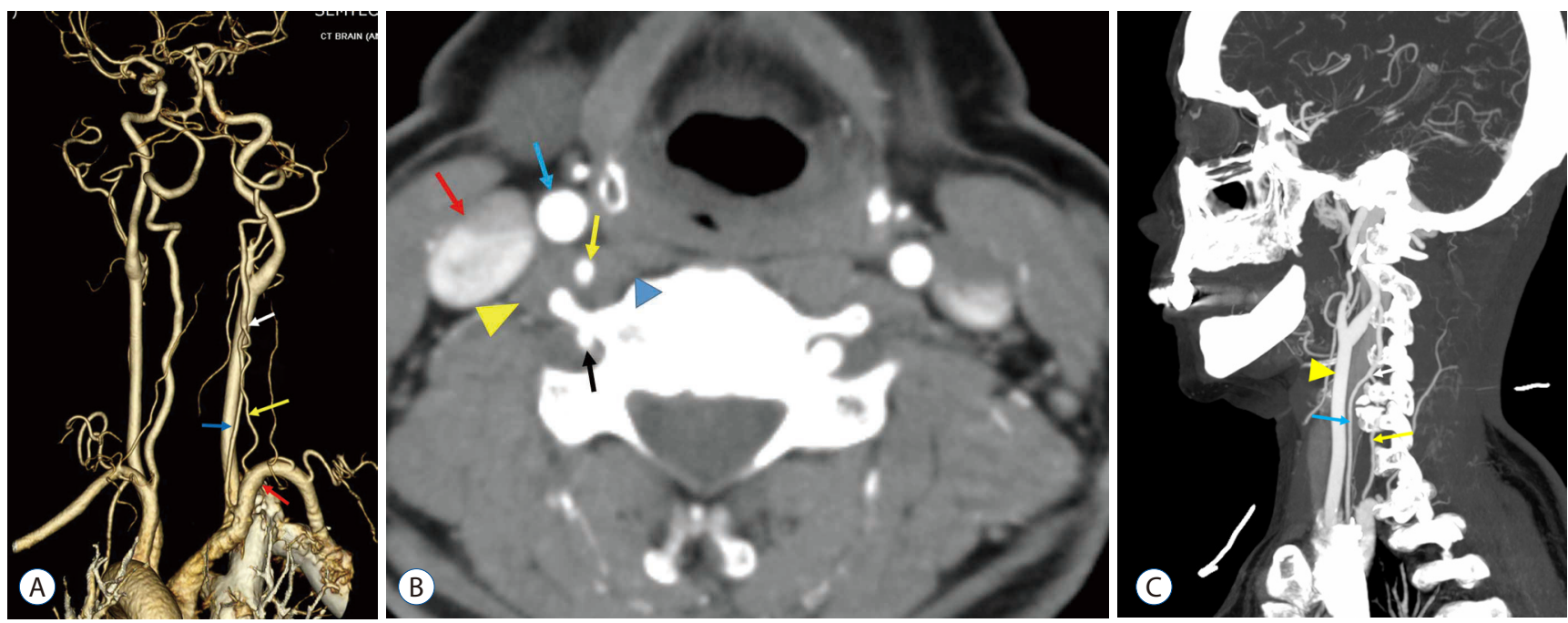

Fig. 3. Computed tomographic (CT) angiography of a 58-year-old woman with a right dual-origin vertebral artery (VA). Reconstructed CT angiography shows a right duplicated VA originating from the right subclavian artery ( $\mathrm{A}$ : red arrow, right subclavian artery; yellow arrow, lateral limb of the duplicated VA; blue arrow, medial limb of the duplicated VA; white arrow, junction of the two limbs of the duplicated VA). Axial neck CT at the fifth cervical vertebral body level after contrast enhancement shows that the medial limb of the duplicated VA is located posteriorly to the right common carotid artery (CCA) and anteriorly to the vertebral transverse foramen. The medial limb of the duplicated VA is located between the longus coli muscle and anterior scalene muscle (B : yellow arrow, medial limb of the right duplicated VA; black arrow, lateral limb of the right VA; blue arrow, right CCA; red arrow, right internal jugular vein; yellow arrowhead, anterior scalene muscle; blue arrowhead, longus coli muscle). Sagittal CT after contrast enhancement shows that the medial limb of the duplicated VA is located in close proximity to the CCA (C: blue arrow, medial limb of the duplicated VA; yellow arrowhead, CCA; yellow arrow, lateral limb of the duplicated VA; white arrow, junction of the two limbs of the duplicated VA).

old man who had a duplication of the right proximal VA and who experienced extensive vertebrobasilar arterial dissection after sexual intercourse. Melki et al. ${ }^{11)}$ reported a 51-year-old man who presented with acute infarction of the cerebellar vermis resulting from dissection of the medial limb of a duplicated right VA after minor neck trauma. Mahmutyazicioğlu et al. ${ }^{9)}$ reported one case of symptomatic thrombosis in one limb of a left duplicated VA. In this patient, electrocardiography revealed paroxysmal atrial tachycardia, which was the most likely explanation for the thrombus. This thrombosis was identified using color Doppler sonography and was treated with anticoagulation, after which the patient's symptoms, including vertigo, weakness, and nausea, ceased.

In a duplicated VA, the other limb may be collateral to the distal VA when the proximal segment of the subclavian artery or the VA originating from the subclavian artery exhibits stenosis $^{2,20)}$. Thrombosis or dissection of one limb of a duplicated VA may result in less severe symptoms compared with other types of VA thrombosis or dissection because of a collateral pathway via another limb of the duplicated VA.

Operative correction of a kinked duplicated origin of the VA, which was suspected to underlie disabling dizziness in a 70-year-old patient, was performed despite the absence of any other evidence of its benefits ${ }^{13)}$. A dual origin of the VA mimicked a dissection on conventional angiography ${ }^{7,14)}$. Polguj et al. ${ }^{15)}$ reported that the lumen of the duplicated VA is smaller than normal; hence, this variant has clinical implications and should be considered when a VA needs catheterization. In such patients, interventional procedures should be performed from the normal side, if possible. Satti et al. ${ }^{18)}$ suggested that a VA can be easily damaged during severe cervical spine injuries with rapid subluxation, deceleration, fracture through the transverse foramen, or flexion of the cervical spine. The additional point of attachment of the VA predisposes individuals with a duplicated origin of the VA to experience more severe outcomes from the kind of contusion that most commonly occurs during motor vehicle accidents.

\section{Potential damage to a duplicated VA during pro- cedures involving the anterior cervical approach and carotid endarterectomy}

Duplication of the VA may be encountered unexpectedly during lower anterior cervical spinal surgery because the entry of the medial limb of the duplicated VA into the vertebral 
transverse foramen is generally higher than usual ${ }^{3,4,8}$. In all 10 patients in this study, the medial limb of the dual-origin VA was located anteriorly and laterally to the cervical transverse foramen, and between the longus coli muscle and anterior scalene muscle. In the anterior spinal approach, the surgeon retracts the longus coli muscle laterally to expose the vertebral body; therefore, the location of the medial limb of the VA should be considered to avoid VA injury. Excessive retraction on the longus coli muscle may lead to injury of the medial limb of the VA. The additional point of attachment of the VA (junction point of both limbs of a duplicated VA) predisposes individuals with a duplicated origin of the VA to suffer from the kind of retraction injury that can occur during the lower cervical anterior approach. During anterior cervical spine operations, the surgeon might select the contralateral approach to avoid damaging the medial limb of a duplicated VA.

In six patients in this study, both limbs of the VA were located in distant position to the CCA and common carotid bifurcation. In the other four patients in this study, the medial limb of the duplicated VA was located in close proximity to the common carotid bifurcation or CCA. In carotid endarterectomy, the surgeon may injure the medial limb of the VA during CCA dissection and proximal clamping if the medial limb of the duplicated VA is located in close proximity to the common carotid bifurcation or CCA. Thus, surgeons should consider this anomaly when performing carotid endarterectomy $y^{4)}$.

\section{CONCLUSION}

Although duplication of the VA is asymptomatic in most cases, clinicians should consider this anomaly during diagnosis and treatment. VA duplication may be encountered unexpectedly during lower cervical anterior surgery and carotid endarterectomy.

\section{PATIENT CONSENT}

The patient provided written informed consent for the publication and the use of their images.

\section{- Acknowledgements}

This work was supported by research grant from National
Medical Center.

\section{References}

1. Dare AO, Chaloupka JC, Putman CM, Mayer PL, Schneck MJ, Fayad PB : Vertebrobasilar dissection in a duplicated cervical vertebral artery: a possible pathoetiologic association? A case report. Vasc Endovascular Surg 31 : 103-109, 1997

2. Eisenberg RA, Vines FS, Taylor SB : Bifid origin of the left vertebral artery. Radiology 159 : 429-430, 1986

3. Giuffrè $R$, Sherkat $S$ : Maldevelopmental pathology of the vertebral artery in infancy and childhood. Childs Nerv Syst 16 : 627-632, 2000

4. Goddard AJ, Annesley-Williams D, Guthrie JA, Weston M : Duplication of the vertebral artery: report of two cases and review of the literature. Neuroradiology $43:$ 477-480, 2001

5. Ionete C, Omojola MF : MR angiographic demonstration of bilateral duplication of the extracranial vertebral artery: unusual course and review of the literature. AJNR Am J Neuroradiol 27 : 1304-1306, 2006

6. Kendi AT, Brace JR : Vertebral artery duplication and aneurysms: 64-slice multidetector CT findings. Br J Radiol 82 : e216-e218, 2009

7. Kim DW : Concomitant dual origin and fenestration of the left vertebral artery resembling dissection. J Korean Neurosurg Soc 46 : 498-500, 2009

8. Komiyama M, Nakajima H, Yamanaka K, Iwai Y : Dual origin of the vertebral artery--case report. Neurol Med Chir (Tokyo) 39 : 932-937, 1999

9. Mahmutyazicioğlu K, Sarac K, Bölük A, Kutlu R : Duplicate origin of left vertebral artery with thrombosis at the origin: color doppler sonography and CT angiography findings. J Clin Ultrasound 26 : 323-325, 1998

10. Meila D, Tysiac M, Petersen M, Theisen O, Wetter A, Mangold A, et al. : Origin and course of the extracranial vertebral artery: CTA findings and embryologic considerations. Clin Neuroradiol 22 : 327-333, 2012

11. Melki E, Nasser G, Vandendries C, Adams D, Ducreux D, Denier C : Congenital vertebral duplication: a predisposing risk factor for dissection. J Neurol Sci 314 : 161-162, 2012

12. Mizukami M, Tomita T, Mine T, Mihara H : Bypass anomaly of the vertebral artery associated with cerebral aneurysm and arteriovenous malformation. J Neurosurg 37 : 204-209, 1972

13. Nishijima M, Harada J, Akai T, Endo S, Takaku A : Operative correction of a kinked duplicate origin of the vertebral artery in a patient with dizziness. Case report. Surg Neurol 32 : 356-359, 1989

14. Nogueira TE, Chambers AA, Brueggemeyer MT, Miller TJ : Dual origin of the vertebral artery mimicking dissection. AJNR Am J Neuroradiol 18 : 382-384, 1997

15. Polguj M, Jędrzejewski K, Topol M, Wieczorek-Pastusiak J, Majos A : Duplication of the left vertebral artery in a patient with dissection of the right internal carotid artery and Ehlers-Danlos syndrome: case report and review of the literature. Anat Sci Int 88 : 109-114, 2013

16. Rameshbabu C, Gupta OP, Gupta KK, Qasim M : Bilateral asymmetrical 
duplicated origin of vertebral arteries: multidetector row CT angiographic study. Indian J Radiol Imaging 24 : 61-65, 2014

17. Rath $G$, Prakash $R$ : Double vertebral artery in an Indian cadaver. Anat Clin 6 : 117-119, 1984

18. Satti SR, Cerniglia CA, Koenigsberg RA : Cervical vertebral artery variations: an anatomic study. AJNR Am J Neuroradiol 28 : 976-980, 2007

19. Sim E, Vaccaro AR, Berzlanovich A, Thaler H, Ullrich CG : Fenestration of the extracranial vertebral artery: review of the literature. Spine (Phila Pa 1976) 26 : E139-E142, 2001

20. Suzuki S, Kuwabara $Y$, Hatano R, Iwai T : Duplicate origin of left vertebral artery. Neuroradiology $15: 27-29,1978$

21. Thomas AJ, Germanwala AV, Vora N, Prevedello DM, Jovin T, Kassam A, et al. : Dual origin extracranial vertebral artery: case report and embryology. J Neuroimaging $18:$ 173-176, 2008 